\title{
Molecularly Imprinted Polymer-Coated Probe Electrospray Ionization Mass Spectrometry Determines Phorbol Esters and Deoxyphorbol Metabolites in Jatropha curcas Leaves
}

\author{
Lidya C. da Silva, ${ }^{1}$ Thays C. de Carvalho, ${ }^{1}$ Igor Pereira, ${ }^{1}$ Julio Cesar Marana, ${ }^{2}$ \\ Bruno G. Laviola, ${ }^{2}$ Patricia V. Abdelnur, ${ }^{2}$ Boniek G. Vaz ${ }^{1} \odot$ \\ ${ }^{1}$ Laboratory of Chromatography and Mass Spectrometry (LaCEM), Chemistry Institute, Federal University of Goiás, Goiânia, GO \\ 74690-900, Brazil \\ ${ }^{2}$ Embrapa Agroenergy, Brazilian Agricultural Research Company, Brasília, DF 70770-901, Brazil
}

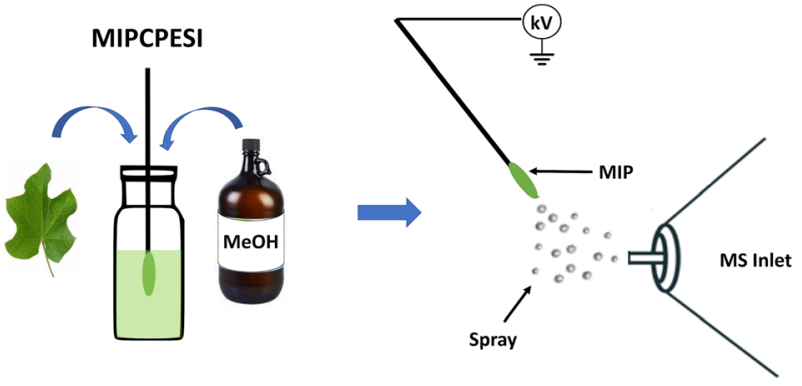

Abstract. In this study, a molecularly imprinted polymer-coated probe electrospray ionization mass spectrometry (MIPCPESI-MS) method was developed for detection of phorbol esters (PEs) and deoxyphorbol metabolites in Jatropha curcas leaves. Such an approach was established by sticking on a metallic needle a molecularly imprinted polymer to particularly design a MIP-coated probe for selective sampling and ionization of PEs and deoxyphorbol metabolites. By a subsequent application of a high voltage and methanol, as spray solvent, ESI was generated for direct and rapid analysis under ambient and open-air conditions. MIP-coated probe exhibited a high sampling capacity of the PEs and its metabolites in methanolic extracts of $\mathrm{J}$. curcas leaves compared with the non-imprinted polymer (NIP)-coated probe. MIPCPESI-MS allowed the detection of phorbol 12,13-diacetate (PDA) from J. curcas leaves with minimal sample preparation, and with detection limit and quantification reaching $0.28 \mu \mathrm{g} / \mathrm{mL}$ and $0.92 \mu \mathrm{g} / \mathrm{mL}$, respectively. Also, good linearity was obtained with $R^{2}>0.99$ and precision and accuracy values between $4.06-13.49 \%$ and -1.60 to $15.26 \%$, respectively. The current method was successfully applied to screening methanolic extracts of six different $J$. curcas leaf genotypes (three toxic and three non-toxic). PDA and three PE deoxyphorbol metabolites were identified only from toxic genotypes, in which PDA was determined with concentration ranging from $222.19 \pm 23.55$ to $528.23 \pm 19.72 \mu \mathrm{g} / \mathrm{g}$. All these findings support that the MIPCPESI-MS method developed here has a high potential for the analysis of PEs in plant extracts enabling differentiation of toxic and non-toxic genotypes earlier in the leaves.

Keywords: Probe electrospray ionization (PESI), Molecularly imprinted polymer (MIP), Phorbol esters

\section{Introduction}

$\mathrm{J}$ atropha curcas L. is an oilseed plant found in tropical and subtropical regions [1]. Its seeds consist of $30-40 \%$ of oil

\footnotetext{
Electronic supplementary material The online version of this article (https:// doi.org/10.1007/s13361-019-02269-5) contains supplementary material, which is available to authorized users.
}

Correspondence to: Boniek Vaz; e-mail: boniek@ufg.br which makes it useful for biodiesel production. After the oil extraction from the seeds, a pinion cake biomass with high protein content $(53-63 \%)$ is produced. This raw material has a high potential for application in animal feed [2]. However, due to the existence of phorbol esters (PEs) on pinion cake, its use for animal feed is not allowed. PEs are toxic compounds that promote tumors, platelet aggregation, apoptosis, cell differentiation, and other adverse metabolic effects [3].

The structure of PEs depends on the tetracyclic diterpene carbon skeleton. Hydroxylation reactions at different positions 
in this basic structure lead to the formation of different structures, as reported for several unstable species of diterpene esters identified from the oil of $J$. curcas seeds [4].

Researches have been conducted to reduce or eliminate the PEs' content from J. curcas. In Brazil, the Brazilian Agricultural Research Company (Embrapa) is using genetic breeding approaches to produce new $J$. curcas genotypes with the absence of toxic compounds, excellent productivity, resistant to pests and diseases, and well-adapted to country's growing conditions $[5,6]$. Traditionally, the differentiation of toxic and non-toxic genotypes is done by detection of PEs in the seeds; therefore, it is necessary that the cultivar reaches the age of seed production. Also, due to the complexity of the samples, extraction and separation techniques are required. Thus, new analytical methods to determine the content of PEs and its metabolites in the unusual matrices as leaves instead of $J$. curcas seed and oil, in order to estimate if the new genotypes are toxic or non-toxic, are highly desirable. Such determination would be advantageous, saving time, because it would not be necessary to wait for the plant growth. For instance, the determination would be made early, in the leaves.

Mass spectrometry (MS) has been widely used in the $J$. curcas analysis for the qualitative and quantitative determination of PEs, as well as in the differentiation of toxic and nontoxic genotypes [7-9]. MS is one of the most sensitive analytical techniques with a wide range of applicability for qualitative and quantitative analysis [10]. As the MS measures only molecules and atoms charged, the ionization process plays an important role in MS [11]. Traditionally, the MS analyses involve ionization techniques under vacuum conditions such as electron ionization (EI), chemical ionization (CI), and matrix-assisted laser desorption ionization (MALDI), as well as atmospheric pressure ionization (API) techniques, such as electrospray ionization (ESI) and atmospheric pressure chemical ionization (APCI) [12]. However, to perform analysis of complex matrixes, sometimes, it is unavoidable the use of laborious sample preparation steps [10].

During the last decade, ionization techniques under ambient conditions have been developed, allowing direct MS analysis with minimum or no sample preparation $[10,12$, 13]. Since the development of desorption electrospray ionization (DESI) and direct analysis in real time (DART), several dozens of new ambient ionization methods have been described [12-14], as extractive electrospray ionization (EESI), rapid evaporative ionization (REIMS), leaf spray, paper spray (PS), and probe electrospray ionization (PESI). All these new ionization methods have been established as prominent methods for rapid and direct analysis under ambient conditions.

Leaf spray is an ambient ionization method applicable to direct detect chemical constituents of intact plant materials [15]. However, the detection of PEs direct from the leaves can be very difficult, mostly due to the lower content of PEs, found to be in concentrations about 1/10 than in the seeds [8].
Hence, in our previous studies, leaf spray was not capable of directly detecting PEs from $J$. curcas leaves [7].

Among ambient ionization methods, PESI has recently attracted attention for being a method derived from ESI with broad applicability and low cost that can be easily assembled in any MS laboratory. PESI was originally developed for liquid sample analysis by Hiraoka et al. [16]. Briefly, a non-capillary needle is used as a sampling probe and ESI emitter under ambient conditions. The needle touches the sample for analyte extraction, and subsequently, the needle is positioned in front of the mass spectrometer inlet orifice. A high voltage is applied to the needle, and an electrolytic spray is formed, followed by the ion analysis by MS. PESI has already been employed for direct MS analysis of foods [17], psychoactive drugs [18], plants [19], metabolites from mouse liver [20], and brain [21], including detection of metabolites at subcellular levels [22].

Despite all advantages, PESI presents some limitations, especially in the analysis of complex samples (e.g., blood, urine, and plants extract), including ionization suppression, low sensitivity at trace level, and narrow dynamic range [12, $23,24]$. In addition, PESI-MS analysis is generally performed using an inert surface needle, which has no enrichment capability. In this regard, it is rather challenging to detect PEs directly and its metabolites from $J$. curcas leaves using the PESI-MS. So, to perform this task, a needle with a high analyte enrichment capability should be developed for PESI-MS.

Integration of sampling, analyte enrichment, and ionization into a single device has emerged as a strategy for highly sensitive analysis in complex matrices using ambient ionization techniques. To date, solid-phase microextraction (SPME)based methods [25] as surface-coated wooden-tip ESI [26] and coated blade spray ionization [27] have been described using such approach for direct and sensitive analysis in complex samples.

Furthermore, polymers may be used as the substrate for MS analyses [28]. Another approach consists of combine molecularly imprinted polymers (MIPs) as an adsorbent to sampling, extraction, and analyte enrichment with ambient ionization methods $[14,29,30]$. MIPs are materials that can provide a chemically selective surface for analyte enrichment. MIP synthesis generates imprinted sites, with similar size and shape, to a target analyte, which are prepared from cross-linking monomers and functional monomers in the presence of a target molecule, known as template $[31,32]$. MIPs have been employed in combination with many ambient ionization methods. For example, MIP was coupled to natural ambient sonic-spray ionization (EASI-MS) for analysis of antipsychotic drugs from urine [33], to wooden-tip electrospray [34] for detection of trace malachite green in aquatic products as well to DESI-MS and paper spray ionization (PSI-MS) for analysis of pesticides from water [35] and fruits [14], respectively.

To improve PESI-MS performance in terms of sensitivity and selectivity, we describe the first example, to the best of our 
knowledge, of using a molecularly imprinted polymer-coated probe electrospray ionization mass spectrometry method for selective analysis of PEs and its metabolites in J. curcas leaf extract. The developed probe, also, to being cost-effective and portable has imprinting cavities of MIPs with specific sites to binding PEs and its metabolites with selective enrichment even at trace levels. Determination of PEs and a series of three deoxy-phorbol metabolites using toxic and non-toxic genotypes of $J$. curcas were realized.

\section{Experimental}

\section{Materials and Reagents}

Leaves of different $J$. curcas genotypes were provided by Embrapa Agroenergy (Brasília, DF). The toxic genotypes were identified as 113F, 199F, and 259F, and the non-toxic genotypes as $169 \mathrm{~F}, 170 \mathrm{~F}$, and $183 \mathrm{~F}$. High-purity chromatographic grade methanol and ethanol were purchased from J. T. Baker (Philipsburg, USA). Formic acid, phorbol-12-myristate-13acetate (PMA), phorbol 12,13-diacetate (PDA), sodium dodecyl sulfate (SDS), tetraethoxysilane (TEOS), and 3aminopropyltriethoxysilane (APTES) were supplied by SigmaAldrich (São Paulo, Brazil). Hydrochloric acid (HCl) was obtained from Scharlab S. L. (Sentmenat, Spain). Deionized water was purified on a Gehaka water-purification system (São Paulo, Brazil). Stained steel rods (4-cm length and diameter of $0.6 \mathrm{~mm}$ ) and resin epoxy were purchased from the local market.

\section{Preparation of Plant Extract}

The leaves were minced into smaller pieces. In sequence, $100 \mathrm{mg}$ of the leaves was placed in an Eppendorf tube with $1 \mathrm{~mL}$ of methanol. The tube was placed into an ultrasonic bath. Extraction was carried out in ambient temperature for $10 \mathrm{~min}$. This extraction procedure was repeated two times for each sample to reach $3 \mathrm{~mL}$ of methanolic extract, which was filtered through filter paper before MS analysis.

\section{Preparation and Characterization of MIP- and NIP-Coated Probe}

For MIP synthesis, $5.9 \mathrm{mmol}$ of TEOS, $12.8 \mathrm{mmol}$ of water, $0.03 \mathrm{mmol}$ of $\mathrm{HCl}$, and $6.8 \mathrm{mmol}$ of ethanol were added to a Falcon tube. The mixture was stirred for $3 \mathrm{~min}$ in a vortex and allowed to stand for $24 \mathrm{~h}$. In sequence, $1.4 \mathrm{mmol}$ of APTES, $3.5 \mathrm{mmol}$ of SDS, and $616.2 \mathrm{mmol}$ of the phorbol 12-myristate 13-acetate were added in another Falcon tube. The mixture was stirred for $3 \mathrm{~min}$ in a vortex, added to the mixture prepared above, and allowed to stand for $24 \mathrm{~h}$.

Posteriorly, the PMA used as template was removed with methanol by Soxhlet extraction using a Sebelin heating battery from Ethiktechnology (São Paulo, Brazil) at $70{ }^{\circ} \mathrm{C}$ for $48 \mathrm{~h}$. To perform the solvent extraction, the imprinted polymers were placed in a paper thimble inside the Soxhlet apparatus; clamps were used to fix the Soxhlet apparatus to the condenser and the extraction flask containing $200 \mathrm{~mL}$ of methanol. After solvent extraction, the polymers were put in the oven at $50{ }^{\circ} \mathrm{C}$ to dry for $6 \mathrm{~h}$; then, they were powdered using porcelain mortar and pestle.

The non-molecularly imprinted polymer (NIP) was also synthesized for purposes of comparing the morphology and performance of the polymers as the extracting phase of the compounds of interest. The NIP synthesis occurred similarly to MIP synthesis without the addition of the template molecule.

Finally, the powdered MIP and NIP were stuck on stained steel needles with $4 \mathrm{~cm}$ length and $0.6 \mathrm{~mm}$ of diameter using a resin epoxy Loctite from Henkel (São Paulo, Brazil). To ensure the evenness, $1 \mathrm{~cm}$ length of the needles were covered with $15 \mathrm{mg}$ of the polymers. The morphology or the MIP and NIP were characterized via scanning electron microscopy (SEM) analysis using a JEOL JSM-6610 (Tokyo, Japan).

\section{Sampling, Extraction, and Mass Spectrometry Analysis}

Mass spectra were acquired on a Q Exactive ${ }^{\mathrm{TM}}$ Hybrid Quadrupole-Orbitrap mass spectrometer (Thermo Scientific, San Jose, USA) and LCQ Fleet (3D ion trap) mass spectrometer (Thermo Scientific, San Jose, USA), recording an appropriate $\mathrm{m} / \mathrm{z}$ in the positive ion mode. Accurate mass measurement was performed using positive ion electrospray Q Exactive Orbitrap with 70,000 resolution power at $\mathrm{m} / \mathrm{z} 200$ using the following parameters: spray voltage of $3.5 \mathrm{kV}$, the capillary temperature of $275^{\circ} \mathrm{C}$, and S-lens RF level of $50 \%$.

For extractive sampling using the MIPCPESI, about $1 \mathrm{~cm}$ of the coated probe was immersed in the methanolic extracts under magnetic stirring for $10 \mathrm{~min}$. After sampling extraction, MIPCPESI probe was washed with ultra-purified water to eliminate other matrix components and then drying at $40{ }^{\circ} \mathrm{C}$ for $30 \mathrm{~min}$.

MIP and NIPCPESI-MS analyses were performed using the LCQ Fleet mass spectrometer (Thermo Scientific, San Jose, USA). MIPCPESI probe was positioned $5 \mathrm{~mm}$ away from the MS inlet at an angle of $105^{\circ}$. To perform ESI, methanol with $0.1 \%$ formic acid was delivered at a flow rate $15 \mu \mathrm{L} / \mathrm{min}$ into MIP-coated surface of the probe, which was connected with a high voltage of $3.5 \mathrm{kV}$ available from the MS. Mass spectra were acquired in positive ion mode with a capillary temperature of $250{ }^{\circ} \mathrm{C}$. Structure confirmation of analytes was performed by tandem mass spectrometry (MS/MS) running collisioninduced dissociation (CID) of $20 \mathrm{eV}$. Helium gas (99.99 purity) was used as the collision gas in the CID experiments.

\section{Analytical Performance}

To PEs quantification, non-toxic $J$. curcas leaf extracts were chosen as matrix, and it was spiked with PDA at different concentration levels $(1,5,10,15,20,25$, and $30 \mu \mathrm{g} / \mathrm{mL})$. Selected reaction monitoring (SRM) mode was used for the quantification experiments (transition of $m / z 431$ to 391). The calibration curve was plotted using the SRM peak about the analyte concentration. The theoretical limit of detection (LOD), representing the smaller amount of the analyte that can be detected, and the limit of quantification (LOQ), representing 
the smaller amount of the analyte that can be determined with acceptable precision and accuracy, were determined from the calibration curve. Theoretical LOD was determined by the following equation $S_{\text {blank }}+3 \times$ oblank, and LOQ was obtained by $S_{\text {blank }}+10 \times \sigma_{\text {blank }}$ where $S_{\text {blank }}$ is the average blank signal and $\sigma_{\text {blank }}$ is the standard deviation of the signal from 10 replicates. Analyses to determine precision, accuracy, and recovery were performed intraday and interday with measurements made in triplicate using three concentrations $(5,15$, and $25 \mu \mathrm{g} / \mathrm{mL}$ ) within the linearity range. Precision was calculated by the equation $\mathrm{CV}=(\mathrm{SD} / \mathrm{AC}) \times 100$, where $\mathrm{CV}$ is the coefficient of variation, $\mathrm{SD}$ is the standard variation, and $\mathrm{AC}$ is the analyzed concentration. Accuracy was calculated by the formula $E=[(\mathrm{AC}-\mathrm{NC}) / \mathrm{NC}] \times 100$, where $E$ is the relative error and $\mathrm{NC}$ is the nominal concentration $[14,36]$. The percentage recovery, $R$, was calculated by the formula $R=(\mathrm{AC} / \mathrm{NC}) \times 100$. Finally, the developed MIPCPESI-MS method was applied for quantification of the phorbol 12,13-diacetate in samples from six different $J$. curcas genotypes.

\section{Results and Discussion}

\section{J. curcas Leaf Extract Characterization}

Routinely, PEs are determined on seeds of $J$. curcas. However, to enable protein-rich $J$. curcas pinion cake in animal feed is necessary to evaluate PEs and its metabolites in other matrices, as the leaves of J. curcas. Such determination would be useful, mainly to save time, on researches that attempt to produce nontoxic genotypes of $J$. curcas. In this way, methanolic extracts from $J$. curcas leaves were analyzed via ESI + Q Exactive mass spectrometer to identify the profile of phorbol esters and its metabolites. As displayed in Fig. 1S, only one PE were identified. The phorbol 12,13-diacetate (PDA) was detected as protonated ion $[\mathrm{M}+\mathrm{H}]^{+}$of $m / z 449.21460$ in all three toxic genotype leaf extracts. Besides the detection of PDA, as displayed in Fig. 2S, a set of deoxyphorbol compounds, derived from phorbol [37], were detected as protonated ions [M+ $\mathrm{H}]^{+}$. These compounds include the 12-deoxyphorbol (I) of $\mathrm{m} / \mathrm{z}$ 347.18530, 4,12-dideoxyphorbol (II) of $\mathrm{m} / \mathrm{z} 329.17474$, and 4,9,12-trideoxyphorbol (III) of $\mathrm{m} / \mathrm{z} 311.16417$.

Phorbol esters are unstable molecules that commonly lose $\mathrm{CH}_{3} \mathrm{COOH}$ and $\mathrm{H}_{2} \mathrm{O}$ groups as reported by Vogg et al. [9]. Fig. $3 \mathrm{~S}$ illustrates a mechanistic proposal explaining the formation of deoxyphorbol derivatives from the phorbol, by removal of oxygen atoms from the parent polyol. However, it remains undetermined if these phorbol analogs are derived from dehydration route or transannular cyclization of less oxygenated lathyrane precursors [38].

\section{MIPCPESI Probe Characterization}

The MIP synthesis includes the formation of the complex between the monomer and the template molecule followed by template removal creating imprinting sites on the polymer surface [32]. Here, aminopropyl triethoxysilane was used as the monomer, tetraethoxysilane as crosslinker reagent, and phorbol 12-myristate 13-acetate was employed as a template in MIP synthesis for PE analysis. The choice of PMA as template was a strategic one that no peaks related to this molecule were detected in the samples previously analyzed by ultra-high-resolution mass spectrometry.

Morphological characterization of the synthesized polymers was performed via scanning electron microscopy (SEM) analysis as illustrated in Figure 1. Note the presence of macroporous layer structure, which covers the stained steel needle surface. According to Cormack and Elorza [39], macroporous polymers are mechanically stable and generally preferable for MIP synthesis. As shown in Figure 1(a), the molecularly imprinted polymer has a higher roughness surface with high porosity and imprinting cavities compared with the non-imprinted polymer stated in Figure 1(b). These results are in agreement with the characterization from other MIPs

\section{(a)}

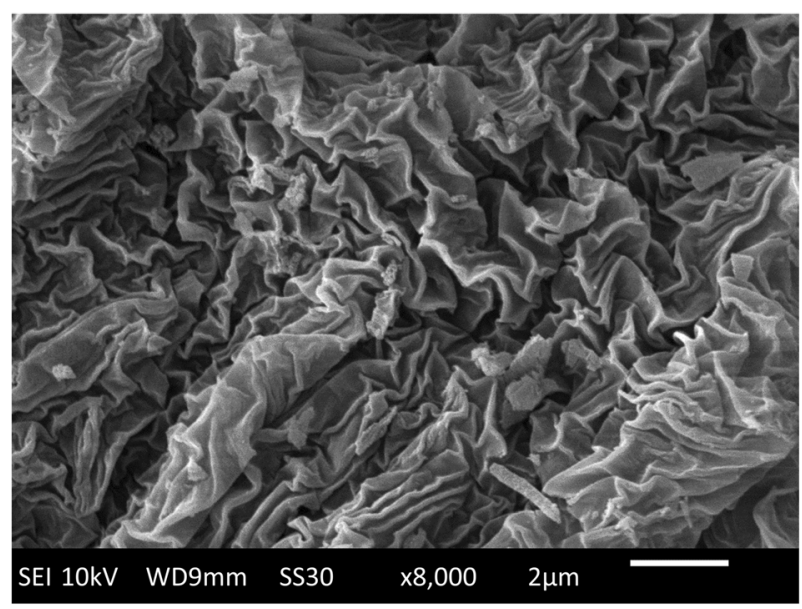

\section{(b)}

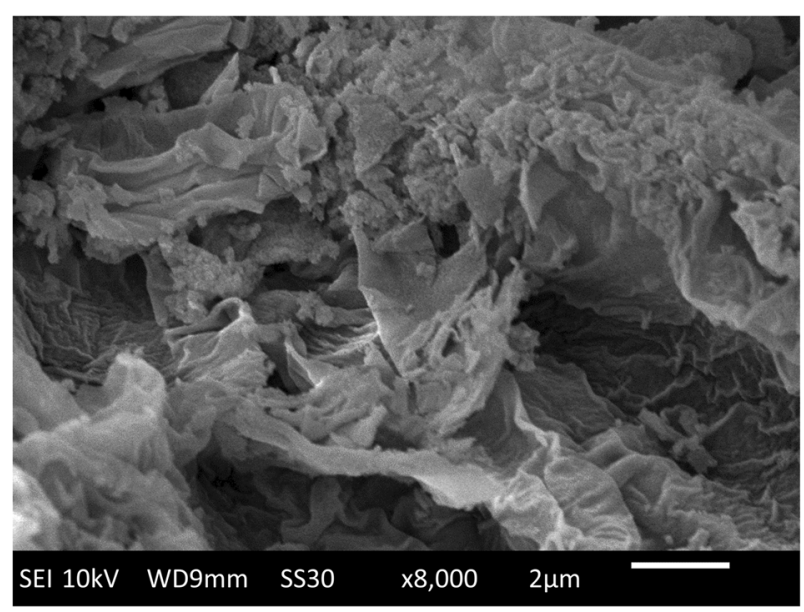

Figure 1. SEM image $(\times 8000)$ of molecularly imprinted polymer (a) and non-imprinted polymer (b) 
synthetized as extraction phase [14, 40, 41].

\section{MIPCPESI-MS Analysis}

As PEs and its phorbol derivatives (e.g., deoxyphorbol) have a common diterpene nucleus which contained different content of hydroxy groups esterified or not, the selective extraction of MIPS on stained steel needle is based on specific sites corresponding to both diterpenic ester and hydroxy group. Methanolic extracted were prepared using $100 \mathrm{mg}$ of toxic $J$. curcas genotype leaves to investigate the analytical performance of MIPCPESI probe in terms of analyte enrichment. The extracted MIPCPESI probe was placed in front of the MS inlet, and $3.5 \mathrm{kV}$ was applied to the probe.
Methanol with 0.1 formic acids was delivered into MIPcoated surface of the probe at a flow rate $15 \mu \mathrm{L} / \mathrm{min}$, as displayed in Figure 2(a). Interestingly, the PDA and the three deoxyphorbol compounds described earlier were identified using MIPCPESI-MS. Figure 2(b) displays the structural formula of detected compounds. As shown in Figure 2(c), the extractive capacity of the MIPCPESI probe was evaluated employing the comparison of the signal intensities obtained in the mass spectra for PDA and three deoxyphorbol detected.

These results demonstrate that the adsorption of the diterpenic phorbol ester group (PDA) is much higher as compared to deoxyphorbol, which contain only diterpenic hydroxy groups (compounds I, II, and III). The data hence suggest that the MIP of MIPCPESI probe is primarily extracting

(a)
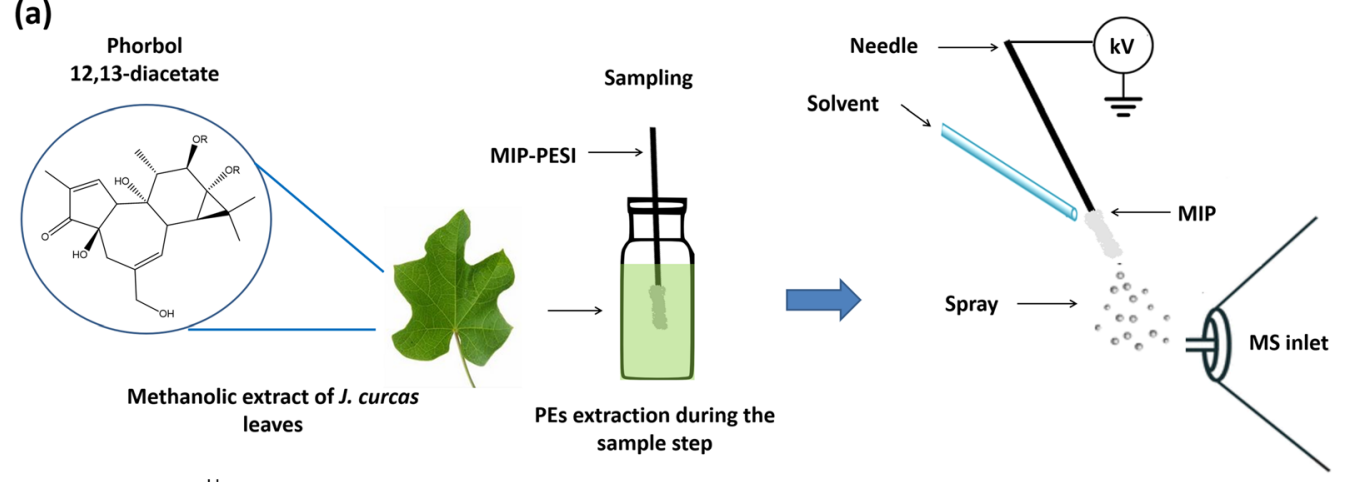

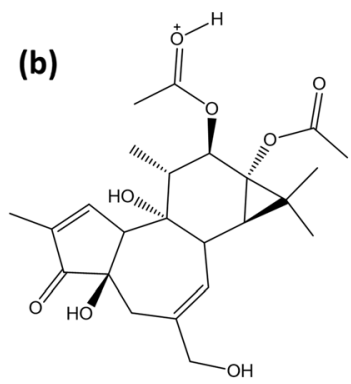

Phorbol 12,13-diacetate

$\left[\mathrm{C}_{24} \mathrm{H}_{32} \mathrm{O}_{8}+\mathrm{H}\right]^{+}$

$\mathrm{m} / \mathrm{z} 449$

(c)

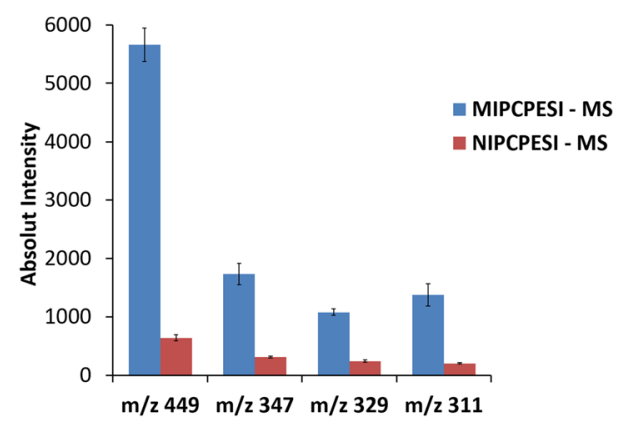

12-deoxyphorbol

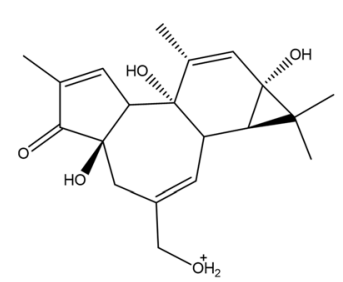
$\left[\mathrm{C}_{20} \mathrm{H}_{26} \mathrm{O}_{5}+\mathrm{H}\right]^{+}$ $\mathrm{m} / \mathbf{2} 347$

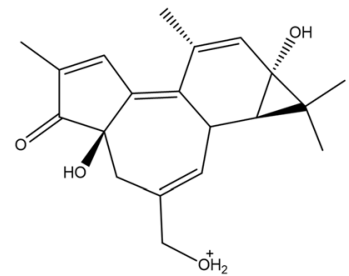

9,12-dideoxyphorbol
$\left[\mathrm{C}_{20} \mathrm{H}_{24} \mathrm{O}_{4}+\mathrm{H}\right]^{+}$ $\mathrm{m} / 2329$

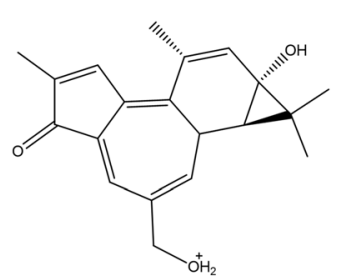

4,9,12-trideoxyphorbol $\left[\mathrm{C}_{20} \mathrm{H}_{24} \mathrm{O}_{4}+\mathrm{H}\right]^{+}$ $\mathrm{m} / \mathrm{z} 311$

(d)

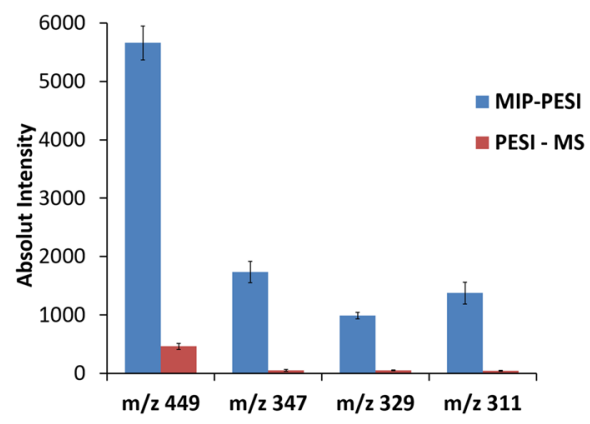

Figure 2. Schematic of the experimental procedure of MIPCPESI-MS analysis (a); structures of the detected phorbol 12,13diacetate, 12-deoxyphorbol, 9,12-dideoxyphorbol, and 4,9,12-trideoxyphorbol (b); bar graph comparing the signal intensities obtained from mass spectra of detect PEs using MIPCPESI-MS and NIPCPESI-MS (c); and comparison between ionization performance of MIPCPESI-MS and traditional PESI-MS (d). Errors bars show the standard deviation of triplicate measurements 
(a)

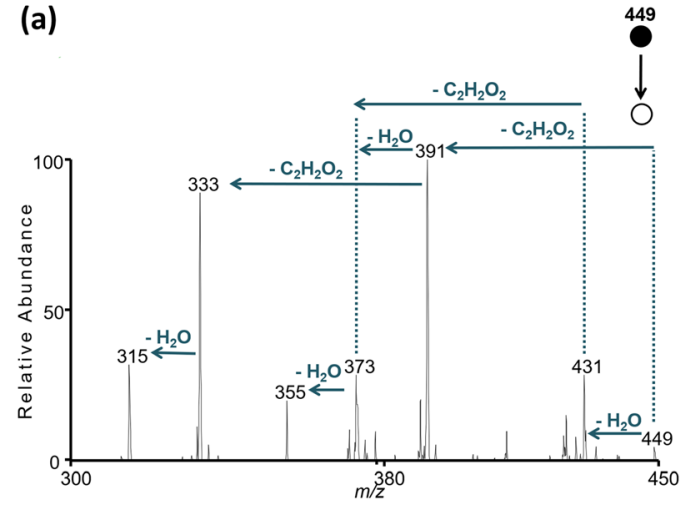

(c)

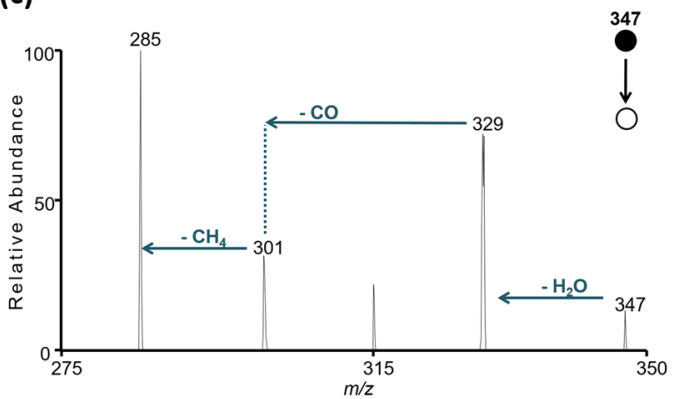

(b)
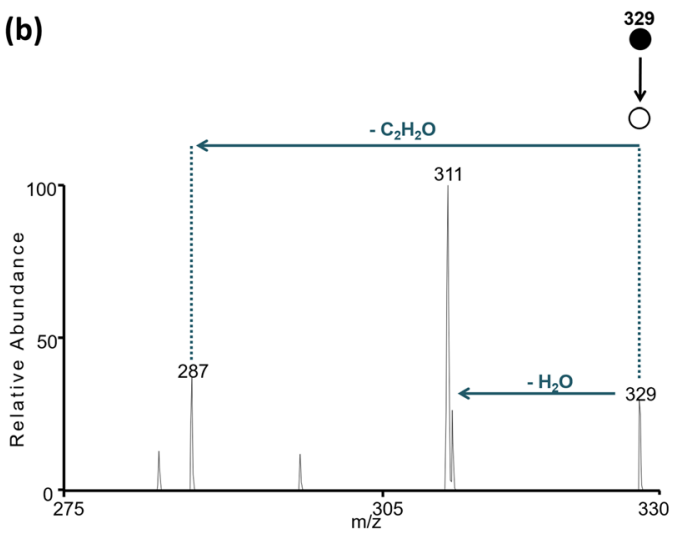

(d)

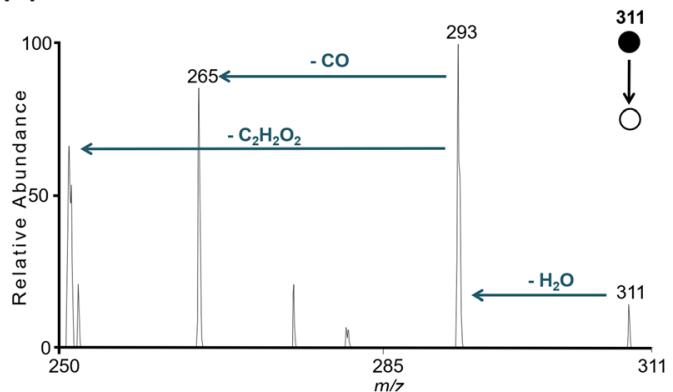

Figure 3. MIPCPESI + MS/MS spectra of the $[\mathrm{M}+\mathrm{H}]^{+}$ions for phorbol 12,13-diacetate (a), 12-deoxyphorbol (b), 4,12-deoxyphorbol (c), and 4,9,12-trideoxyphorbol (d)

compounds with diterpenic phorbol moiety, mainly PDA. This experimental result was expected due to the more significant similarity of the PDA to the PMA, used as a template for design imprinting sites in polymer, compared with the similarity between PMA and deoxyphorbol compounds. MIP selectivity is based on its ability to recognize and re-bind the template molecule [31, 42, 43].

The comparison between the NIP and MIPCPESI-MS analysis in Figure 2(c) corroborates with a previously discussed statement. MIPCPESI-MS provides higher signal intensities

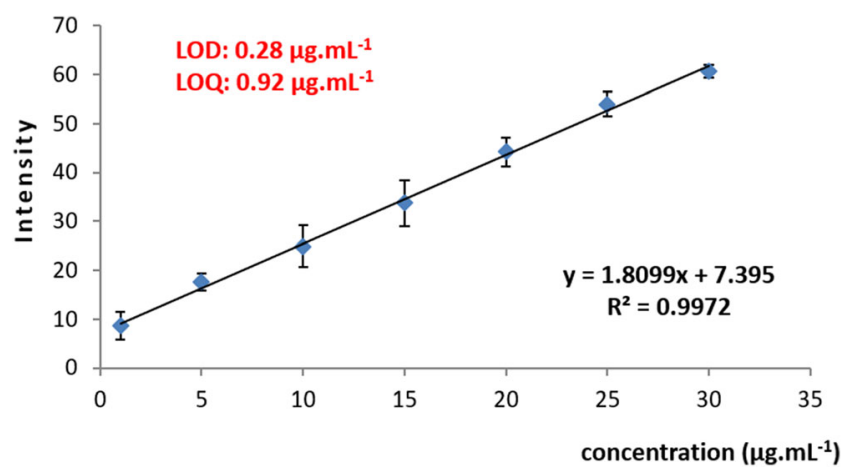

Figure 4. The calibration curve, theoretical LOD, and LOQ obtained via MIPCPESI + MS/MS analyses of the blank matrix with PDA addition in concentrations ranging 1 to $30 \mu \mathrm{gL}^{-1}$. Error bars show the standard deviation of measurements made in triplicate for the peaks related to phorbol ester and deoxyphorbol compounds in comparison with NIPCPESI-MS. Imprinted cavities of MIPCPESI probe favors PEs and phorbol analog extraction, leading us to improve the selective and specific extraction of such compounds at trace levels from J. curcas leaf extract.

Fig. 4S displays the positive ion mode mass spectra of methanolic extracts of toxic $J$. curcas genotype leaves obtained by MIPCPESI probe and by the conventional PESI probe (stained steel needle). As discussed above, MIPCPESI probe allowed the detection of PDA and phorbol analogs and deoxyphorbol compounds. On the other hand, when the methanolic extract of toxic $J$. curcas leaves was interrogated by conventional PESI probe, only PDA $(\mathrm{m} / \mathrm{z} 449)$ was detected at low abundance. The ions of $m / z 347,329$ and 311, referent to deoxyphorbol compounds I, II, and III, respectively, were not detected. As mentioned earlier, the plant extract is a complex matrix, and its composition might cause a deleterious effect on the ionization process. These components can suppress analyte signal, causing it to be cloaked by interferent ions, thus equally lowering sensitivity and complicating the spectrum. As MIPCPESI probe integrates both analyte enrichment and ionization capability, it is less susceptible to ionic suppression inherent of the matrix sample compared with the non-coated stained steel needle used in conventional PESI. As noted in the bar chart shown in Figure 2(d), MIPCPESI probe possessed excellent extraction capacity for analysis of methanolic $J$. curcas leaf extract compared to conventional PESI. 
Table 1. Precision, Accuracy, and Recovery for MIPCPESI Method in the Analysis of Phorbol 12,13-Diacetate in J. curcas Leaf Extracts

\begin{tabular}{|c|c|c|c|c|}
\hline \multirow[t]{5}{*}{ Intraday } & $\mathrm{NC}^{\mathrm{a}}\left(\mu \mathrm{g} \mathrm{mL}^{-1}\right)$ & 5 & 15 & 25 \\
\hline & $\mathrm{AC}^{\mathrm{b}}\left(\mu \mathrm{g} \mathrm{mL}^{-1}\right) \pm \mathrm{UC}^{\mathrm{c}}(\%)$ & $4.92 \pm 1.12$ & $13.24 \pm 1.72$ & $27.68 \pm 4.37$ \\
\hline & Precision $\left(\mathrm{CV}^{\mathrm{d}} \%\right)$ & 13.49 & 7.72 & 9.37 \\
\hline & Accuracy $E^{\mathrm{e}} \%$ & -1.60 & -11.70 & 10.73 \\
\hline & Recovery \% & 98.40 & 88.27 & 110.72 \\
\hline \multirow[t]{5}{*}{ Interday } & $\mathrm{NC}^{\mathrm{a}}\left(\mu \mathrm{g} \mathrm{mL}^{-1}\right)$ & 5 & 15 & 25 \\
\hline & $\mathrm{AC}^{\mathrm{b}}\left(\mu \mathrm{g} \mathrm{mL}^{-1}\right) \pm \mathrm{UC}^{\mathrm{c}}(\%)$ & $5.65 \pm 0.74$ & $12.71 \pm 0.93$ & $26.41 \pm 1.81$ \\
\hline & Precision $\left(\mathrm{CV}^{\mathrm{d}} \%\right)$ & 7.78 & 4.35 & 4.06 \\
\hline & Accuracy $E^{\mathrm{e}} \%$ & 12.77 & -15.26 & 5.65 \\
\hline & Recovery \% & 113.00 & 84.73 & 105.64 \\
\hline
\end{tabular}

\footnotetext{
${ }^{\mathrm{a} N o m i n a l ~ c o n c e n t r a t i o n ~}$

${ }^{\mathrm{b}}$ Analyzed concentration

${ }^{c}$ Expanded uncertainty $(k=2$ for $P=95 \%)$

${ }^{\mathrm{d}}$ Coefficient of variation

${ }^{\mathrm{e}}$ Relative error
}

MIPCPESI-MS is suitable for the determination of trace level of PEs and phorbol analogs in J. curcas leaves.

As MIPCPESI mass spectra were acquired on LCQ mass spectrometer, PDA and deoxyphorbol ions were subjected to the collision-induced dissociation (CID) experiments to confirm their identities. Figure 3 shows the MIPCPESI + MS/MS spectra of $[\mathrm{M}+\mathrm{H}]^{+}$ions for PDA and deoxyphorbol molecules.

In Figure 3(a), CID of the $m / z 449$ corroborates to phorbol 12,13-diacetate, exhibiting characteristics loss of water (18 Da) molecules and acetate groups present in their structure observed in different fragmentation routes. The PDA molecule has several ionization sites; therefore, many fragmentation routes are possible. Fig. 5S shows some possible fragmentation routes from ionization in one of the esters groups (a) and ionization in one of the hydroxyl groups (b).

In CID spectra, the 12-deoxyphorbol, 4,12-deoxyphorbol, and 4,9,12-deoxyphorbol, respectively shown in Figure 3(a), (b), and (c), exhibited neutral losses of $\mathrm{H}_{2} \mathrm{O}$ (18 Da) molecules. The dihydroxylation is commonly observed in phorbol structures [4], as observed in CID spectra; the elimination of water molecules brings stability and abundance to the resulting fragment ions. In addition to removal of hydroxyl groups, for the $m / z$ 347 occurs the elimination of the ketone group $(\mathrm{C}=\mathrm{O}, 28 \mathrm{Da})$ (see fragmentation mechanism proposed in Fig. 6S). For the 4,12-dideoxyphorbol of $m / z 329$, the elimination of one water molecule generates the ion of $\mathrm{m} / \mathrm{z} 311$ commonly found in samples containing PEs since it is related to the main degradation product of the phorbol nucleus [8,9]. In a second fragmentation route, via a rearrangement $[1,3], \mathrm{H}$ occurs the elimination of ethanol group $(\mathrm{HC} \equiv \mathrm{COH}, 42 \mathrm{Da})$. The proposed fragmentation mechanisms from ionization in two different sites are shown in Fig. 7S. In CID spectrum, the ion 4,9,12-trideoxyphorbol $(\mathrm{m} / \mathrm{z}$ 311) exhibit typical fragmentation pattern obtained by removal of a water molecule followed by the loss of a ketone group consisting with the commonly reported in the literature (see fragmentation mechanism proposed in Fig. 8S).

\section{MIPCPESI Probe Analytical Performance}

Quantitative analysis of the phorbol 12,13-diacetate $(\mathrm{m} / \mathrm{z} 449)$ in methanolic extracts of $J$. curcas leaves achieved a calibration curve constructed with samples of the blank matrix with addition of PDA standard in seven concentrations ranging from 1 to $30 \mu \mathrm{g} / \mathrm{mL}$. According to Baldini et al. [8], the expected concentration of PEs in J. curcas leaves is approximately $525 \mu \mathrm{g} / \mathrm{g}$. Therefore, the working range was chosen for the $100 \mathrm{mg}$ of leaves used in the preparation of $3 \mathrm{~mL}$ of samples in which an aliquot of $1 \mathrm{~mL}$ was analyzed [8]. The SRM (single reaction monitoring) mode was used to monitor the transition of $\mathrm{m} / \mathrm{z} 449$ to 391 in the function of the concentration of PDA standard in the samples. Fragmentation spectra used to build the analytical curve are shown in Fig. 9S.

Using MIPCPESI-MS, the signal intensities ratios of the most abundant MS/MS transitions were found to be linear $(y=$ $\left.1.8099 x+7.395, R^{2}=0.9972\right)$. Theoretical LOD and LOQ were calculated from the calibration curve, resulting, respectively, in values of 0.28 and $0.92 \mathrm{ppm}$ as shown in Figure 4. The LOD and LOQ values found in the $\mu \mathrm{g} / \mathrm{mL}$ range are in

Table 2. Concentrations of Phorbol 12,13-Diacetate Determined in J. curcas via Analysis MIPCPESI + MS/MS

\begin{tabular}{|c|c|}
\hline Sample & PDA concentration $\left(\mu \mathrm{g} \mathrm{mL}^{-1}\right) \pm \mathrm{UC}^{\mathrm{a}}(\%)$ \\
\hline $113 \mathrm{~F}$ & $344.85 \pm 20.27$ \\
\hline $199 \mathrm{~F}$ & $222.19 \pm 23.55$ \\
\hline $259 \mathrm{~F}$ & $528.23 \pm 19.72$ \\
\hline $170 \mathrm{~F}$ & $<\mathrm{LOD}^{\mathrm{b}}$ \\
\hline $171 \mathrm{~F}$ & $<\mathrm{LOD}^{\mathrm{b}}$ \\
\hline $183 \mathrm{~F}$ & $<\mathrm{LOD}^{\mathrm{b}}$ \\
\hline
\end{tabular}

${ }^{\mathrm{a}}$ Expanded uncertainty $(K=2$ for $P=95 \%)$

${ }^{\mathrm{b}} \mathrm{LOD}=0.28 \mu \mathrm{g} \mathrm{g}^{-1}$ 
agreement with results reported in the literature for PEs' determination in leaves and seeds of $J$. curcas via analyses HPLCMS/MS and HPLC-UV $[8,44]$.

Analyses to determine precision and accuracy were done in triplicate at low (5 ppm), medium (15 ppm), and high $(25 \mathrm{ppm})$ concentration levels contemplating the linearity range. For intraday analyses, recovery values between 88.27 and $110.72 \%$ are found, also, the lowest precision value was $7.72 \%$ for the sample at $15 \mu \mathrm{g} / \mathrm{mL}$, and the lowest accuracy value was $-1.60 \%$ at $5 \mu \mathrm{g} / \mathrm{mL}$. For interday analyses, the lowest values were obtained for the sample at $25 \mu \mathrm{g} / \mathrm{mL}$ of $4.35 \%$ for precision and $5.65 \%$ for accuracy, while the recovery was found between 84.73 and $113.00 \%$. Table 1 shows all precision and accuracy values obtained for MIPCPESI method. The presented coefficients of variation and relative errors lower than $20 \%$ are established in the Brazilian Manual of the Ministry of Agriculture, Livestock, and Supply (MAPA). Moreover, these results with precision and accuracy close to $15 \%$ are in agreement with those reported in the literature using MIP and ambient mass spectrometry [14, 33].

Posteriorly, MIPCPESI method was employed in the determination of phorbol 12,13-diacetate in methanolic extracts of $J$. curcas leaves. The genotypes analyzed were yielded by Embrapa Agroenergia (Brasília, Brazil). The results reported in Table 2 show the content of PDA determined in toxic (113F, $199 \mathrm{~F}$, and 259F) and non-toxic (170F, 171F, and 183F) genotypes. The sample $259 \mathrm{~F}$ presented the highest concentration of PDA, and the lowest concentration was found for samples 199F. No peak related to phorbol esters were detected in nontoxic leaves.

\section{Conclusions}

In this work, the practicality of the ionization by probe electrospray was combined with the selectivity of the MIP used as extraction phase in the determination of PEs in leaf extracts of $J$. curcas. The MIP-coated probe proved to be adequate for extraction of the PEs in methanolic extracts of $J$. curcas leaves with better performance as extraction phase in comparison with the coated non-imprinted polymer. We have demonstrated MIPCPESI as an effective method to perform an extraction with lower solvent demand and effective ionization for detection of a target molecule in a complex matrix using an ambient mass spectrometry technique. The method presented linearity with $R^{2}$ greater than 0.99, theoretical LOD and LOQ in the ppm range, and satisfactory values of accuracy and precision lower than $20 \%$. The method was applied to determine the contents of PDA in methanolic extracts of $J$. curcas leaves; no content of PEs was detected in non-toxic genotypes; for the toxic, concentrations ranging from $222.19 \pm 23.55$ to $528.23 \pm 19.72 \mu \mathrm{g} /$ $\mathrm{g}$ were determined. Therefore, the presented method is promising to be optimized and applied for other analytes and matrices.

\section{Acknowledgements}

This work received institutional and financial support by CAPES, CNPq, and FAPEG.

\section{References}

1. Nakao, M., Hasegawa, T., Yusuhara, T., Ishihara, Y.: Degradation of Jatropha curcas phorbol esters derived from Jatropha oil cake and their tumor promoting activity. Ecotox. Environ. Safe. 114, 357-364 (2015)

2. Hass, W., Sterk, H., Mittelbach, M.: Novel 12-deoxy-16-hydroxyphorbol diesters isolated from the seed oil of Jatropha curcas. J. Nat. Prod. 65, 1435-1440 (2002)

3. Devappa, R.K., Makkar, H.P.S., Becker, K.: Optimization of conditions for the extraction of phorbol esters from Jatropha oil. Biomass Bioenergy. 34, 1125-1133 (2010)

4. Goel, G., Makkar, H.P.S., Francis, G., Becker, K.: Phorbol esters: structure, biological activity and toxicity in animals. Int. J. Toxicol. 26, 279288 (2007)

5. Laviola, B.G., Teodoro, P.E., Peixoto, L.A., Bhering, L.L.: Parental selection in diallel crosses of Jatropha curcas using mixed models. Acta Sci. 40, 51-57 (2018)

6. Laviola, B.G., Alves, A.A., Rosado, T.B., Bhering, L.L., Formighieri, E.F., Peixoto, L.A.: Establishment of new strategies to quantify and increase the variability in the Brazilian Jatropha genotypes. Ind. Crop. Prod. 117, 216-233 (2018)

7. Pereira, I., Carvalho, T.C., Romão, W., Filgueiras, P.R., Laviola, B.G., Rodrigues, C.M., Abdelnur, P.V., Vaz, B.G.: Differentiation of toxic and non-toxic leaves of Jatropha curcas L. genotypes by leaf spray mass spectrometry. J. Brz. Chem. Soc. 28, 1461-1466 (2017)

8. Baldini, M., Ferfuia, C., Bortolomezzi, R., Verardo, G., Pascali, J., Piasentier, E., Franceschi, L.: Determination of phorbol esters in seeds and leaves of Jatropha curcas and in animal tissue by high-performance liquid chromatography tandem mass spectrometry. Ind. Crop. Prod. 59, 268-276 (2014)

9. Vogg, G., Achatz, S., Kettrup, A., Sandermann Jr., H.: Fast, sensitive and selective liquid chromatographic-tandem mass spectrometric determination of tumor-promoting diterpene esters. J. Chromatogr. A. 855, 563573 (1999)

10. Ma, X., Ouyang, Z.: Ambient ionization and miniature mass spectrometry system for chemical and biological analysis. TRAC. 85, 10-19 (2016)

11. Cooks, R.G., Ouyang, Z., Takats, Z., Wiseman, J.: Ambient mass spectrometry. Science. 311, 1566-1570 (2006)

12. Ouyang, Z., Zhang, X.: Ambient mass spectrometry. Analyst. 135, 659$660(2010)$

13. Huang, M., Yuan, C., Cheng, S., Cho, S., Shie, J.: Ambient ionization mass spectrometry. Annu. Rev. Anal. Chem. 3, 43-65 (2010)

14. Pereira, I., Rodrigues, M.F., Chaves, A.R., Vaz, B.G.: Molecularly imprinted polymer (MIP) membrane assisted direct spray ionization mass spectrometry for agrochemicals screening in foodstuffs. Talanta. 178, 507-514 (2018)

15. Liu, J., Wang, H., Cooks, R.G., Ouyang, Z.: Leaf spray: direct chemical analysis of plant material and living plants by mass spectrometry. Anal. Chem. 83, 7608-7613 (2011)

16. Hiraoka, K., Nishidate, K., Mori, K., Asakawa, D., Suzuki, S.: Development of probe electrospray using a solid needle. Rapid Commun. Mass Spectrom. 21, 3139-3144 (2007)

17. Usmanov, D.T., Mandal, M.K., Hiraoka, K., Ninomiya, S., Wada, H., Matsumura, M., Sanada-Morimura, S., Nonami, H., Yamabe, S.: Dipping probe electrospray ionization/mass spectrometry for direct on-site and low-invasive food analysis. Food Chem. 260, 53-60 (2018)

18. Usui, K., Murata, T., Fujita, Y., Kamijo, Y., Hanazawa, T., Yoshizawa, T., Funayama, M.: Direct detection of the psychoactive substance MT-45 in human tissue samples by probe electrospray ionization-tandem mass spectrometry. Drug Test Anal. 10, 1033-1038 (2018)

19. Liu, J., Xia, B., Ji, B., Xiao, S., Ding, L., Zhou, Y.: Strategy to rapidly discriminate trace isomeric lignan compounds from Gymnotheca chinensis by probe electrospray ionization tandem mass spectrometry. Eur. J. Mass Spectrom. 21, 37-44 (2015)

20. Zaitsu, K., Hayashi, Y., Murata, T., Ohara, T., Nakagiri, K., Kusano, M., Nakajima, H., Nakajima, T., Ishikawa, T., Tsuchihashi, H., Ishii, A.: 
Intact endogenous metabolite analysis of mice liver by probe electrospray ionization/triple quadrupole tandem mass spectrometry and its preliminary application to in vivo real-time analysis. Anal. Chem. 88, 3556-3561 (2016)

21. Zaitsu, K., Hayashi, Y., Murata, T., Yokota, K., Ohara, T., Kusano, M., Tsuchihashi, H., Ishikawa, T., Ishii, A., Ogata, K., Tanihata, H.: In vivo real-time monitoring system using probe electrospray ionization/tandem mass spectrometry for metabolites in mouse brain. Anal. Chem. 90, 4695-4701 (2018)

22. Gong, X., Zhao, Y., Cai, S., Fu, S., Yang, C., Zhang, S., Zhang, X.: Single cell analysis with probe ESI-mass spectrometry: detection of metabolites at cellular and subcellular levels. Anal. Chem. 86, 3809-3816 (2014)

23. Alberici, R.M., Simas, R.C., Sanvido, G.B., Romão, W., Lalli, P.M., Benassi, M., Cunha, I.B.S., Eberlin, M.N.: Ambient mass spectrometry: bringing MS into the "real world". Anal. Bioanal. Chem. 398, 265-294 (2010)

24. Chen, L.C., Nishidate, K., Salto, Y., Mori, K., Asakawa, D., Takeda, S., Kubota, T., Hori, H., Hiraoka, K.: Characteristics of probe electrospray generated from a solid needle. J. Phys. Chem. B. 112, 11164-11170 (2008)

25. Gómez-Ríos, G.A., Liu, C., Tascon, M., Reyes-Garcés, N., Arnold, D.W., Covey, T.R., Pawliszyn, J.: Open port probe sampling interface for the direct coupling of biocompatible solid-phase microextraction to atmospheric pressure ionization mass spectrometry. Anal. Chem. 89, 38053809 (2017)

26. Deng, J., Yu, T., Yao, Y., Peng, Q., Luo, L., Chen, B., Wang, X., Yang, Y., Luan, T.: Surface-coated wooden-tip electrospray ionization mass spectrometry for determination of trace fluoroquinolone and macrolide antibiotics in water. Anal. Chim. Acta. 954, 52-59 (2017)

27. Tascon, M., Gomez-Rios, G.A., Reyes-Garces, N., Poole, J., Boyaci, E., Pawliszyn, J.: High-throughput screening and quantification of target compounds in biofluids by coated blade spray-mass spectrometry. Anal. Chem. 89, 8421-8428 (2017)

28. Song, X., Chen, H., Zare, R.N.: Conductive polymer spray ionization mass spectrometry for biofluid analysis. Anal. Chem. 90, 12878-12885 (2018)

29. Tavares, L.S., Carvalho, T.C., Romão, W., Vaz, B.G., Chaves, A.R.: Paper spray tandem mass spectrometry based on molecularly imprinted polymer substrate for cocaine analysis in oral fluid. J. Am. Soc. Mass Spectrom. 29, 566-572 (2018)

30. Mendes, T.P.P., Pereira, I., Ferreira, M.R., Chaves, A.R., Vaz, B.G.: Molecularly imprinted polymer-coated paper as a substrate for highly sensitive analysis using paper spray mass spectrometry: quantification of metabolites in urine. Anal. Methods. 43, 6117-6123 (2017)
31. Murray, A., Örmeci, B.: Application of molecularly imprinted and nonimprinted polymers for removal of emerging contaminants in water and wastewater treatment: a review. Environ. Sci. Pollut. Res. 19, 3820-3830 (2012)

32. Salehi, S., Rasoull-Amini, S., Adib, N., Shekarchi, M.: Synthesis of molecular imprinted polymers for selective extraction of domperidone from human serum using high performance liquid chromatography with fluorescence detection. J. Chromatogr. B. 1027, 165-173 (2016)

33. Figueiredo, E.C., Sanvido, G.B., Arruda, M.A.Z., Eberlin, M.N.: Molecularly imprinted polymers as analyte sequesters and selective surfaces for easy ambient sonic-spray ionization. Analyst. 135, 726-730 (2010)

34. Huang, Y., Ma, Y., Hu, H., Guo, P., Miao, L., Yang, Y., Zhang, M.: Rapid and sensitive detection of trace malachite green and its metabolite in aquatic products using molecularly imprinted polymer-coated woodentip electrospray ionization mass spectrometry. RSC Adv. 7, 52091-52100 (2017)

35. Biesen, G.V., Wiseman, J.M., Li, J., Bottaro, C.S.: Desorption electrospray ionization-mass spectrometry for the detection of analytes extracted by thin-film molecularly imprinted polymers. Analyst. 135, 2237-2240 (2010)

36. Thompson, M., Ellison, S.L.R., Wood, R.: Harmonized guidelines for single-laboratory validation of methods of analysis (IUPAC Technical Report). Pure App. Chem. 74, 835-855 (2002)

37. Wang, H.-B., Wang, X.-Y., Liu, L.-P., Qin, G.-W., Kang, T.-G.: Tigliane diterpenoids from the Euphorbiaceae and Thymelaeaceae families. Chem. Rev. 115, 2975-3011 (2015)

38. Appendino, G., Belloro, E., Tron, G.C., Jakupovic, J., Ballero, M.: Diterpenoids from Euphorbia pithyusa subsp. Cupanii. J. Nat. Prod. 62, 1399-1404 (1999)

39. Cormack, P.A.G., Elorza, A.Z.: Molecularly imprinted polymers: synthesis and characterization. J. Chromatogr. B. 804, 173-182 (2004)

40. Krupadam, R.J., Khan, M.S., Wate, S.R.: Removal of probable human carcinogenic polycyclic aromatic hydrocarbons from contamined water using molecularly imprinted polymer. Water Res. 44, 681-688 (2010)

41. Zhang, Z., Hu, J.: Effect of environmental factors on estrogenic compounds adsorption by MIP. Water Air Soil Pollut. 210, 255-264 (2010)

42. Anene, A., Hosni, K., Chevalier, Y., Kalfat, R., Hbaieb, S.: Molecularly imprinted polymer for extraction of patulin in apple juice samples. Food Control. 70, 90-95 (2016)

43. Capriotti, A.L., Cavaliere, C., Giansanti, P., Gubbiotti, R., Samperi, R., Laganà, A.: Recent developments in matrix solid-phase dispersion extraction. J. Chromatogr. A. 1271, 2521-2532 (2008)

44. Devappa, R.K., Bingham, J.P., Khanal, S.K.: High performance liquid chromatography method for rapid quantification of phorbol esters in Jatropha curcas seed. Ind. Crop. Prod. 49, 211-219 (2013) 